
\title{
28 Research Square \\ Relationship Between Indoor Solid Fuel Use for Cooking with Blood Pressure and Hypertension Among the Elderly in China
}

Qiutong Yu

Shandong University School of Medicine: Shandong University Cheeloo College of Medicine https://orcid.org/0000-0002-1758-0692

Genyong Zuo ( $\nabla$ smartyong@sdu.edu.cn )

Shandong University School of Medicine: Shandong University Cheeloo College of Medicine https://orcid.org/0000-0002-9305-9671

\section{Research Article}

Keywords: indoor solid fuel, blood pressure, epidemiology, hypertension, indoor air pollution, global health

Posted Date: January 18th, 2022

DOI: https://doi.org/10.21203/rs.3.rs-1166654/v1

License: (c) (i) This work is licensed under a Creative Commons Attribution 4.0 International License.

Read Full License

Version of Record: A version of this preprint was published at Environmental Science and Pollution Research on March 14th, 2022. See the published version at https://doi.org/10.1007/s11356-022-196121. 


\section{Abstract}

Indoor air pollution caused by solid fuel cooking use affects 2.5 billion people worldwide and may elevate blood pressure (BP) and increase the burden of hypertension. Although the elderly are the most at risk of elevated BP and hypertension, few studies have evaluated the effect of indoor solid fuel use for cooking on BP in persons over the age of 65 . Therefore, in this study, we randomly selected 8067 elderly people aged over 65 years from the 2018 Chinese Longitudinal Healthy Longevity Survey to determine the impact of indoor solid fuel use on BP/hypertension. The results showed that, compared with those who cooked with clean fuel, those who cooked with solid fuel had a $1.87 \mathrm{mmHg}$ higher systolic blood pressure, a $0.09 \mathrm{mmHg}$ higher diastolic blood pressure, a $0.97 \mathrm{mmHg}$ higher pulse pressure, and a 1.22 $\mathrm{mmHg}$ higher mean arterial pressure. However, we did not find any association between indoor solid fuel use and hypertension. We further observed that northern China residents, women, people aged over 90 years, hypertensive and heart patients, and those with natural ventilation in the kitchen that used indoor solid fuel experienced a greater BP impact. Replacing solid fuel with clean fuel may be an important way to lower BP. Regarding this, priority access to clean fuels should be given to the susceptible population, including the elderly aged $\geq 75$ years, northern China residents, women, and hypertensive and heart patients.

\section{Introduction}

Air quality is an important determinant of human health (Brauer et al., 2021). According to the World Health Organization, approximately $91 \%$ of the world's population breathes polluted air. Air pollution is a man-made disaster that seriously affects the quality of human life and disability adjusted life years (Cohen et al., 2017). It is estimated that environmental air pollution causes 4.2 million deaths each year as a result of strokes, heart disease, lung cancer, and chronic respiratory diseases (World Health Organization, 2021). Among these mortalities, nearly 2.8 million people died as a result of indoor air pollution caused by the use of solid fuel (Cohen et al., 2017).

In 2010, indoor air pollution from solid fuel use negatively impacted the global disease burden (Lim et al., 2012). Globally, approximately 2.5 billion people have been exposed to indoor air pollution from cooking with solid fuel (Bonjour et al., 2013). Burning solid fuel such as coal, wood, or crop residues can produce a large amount of particulate matter $\left(\mathrm{PM}_{2.5}\right.$ and $\left.\mathrm{PM}_{10}\right)$, carbon monoxide, nitrogen dioxide, sulfur dioxide, and other volatile organic compounds, which are associated with blood pressure (BP) and hypertension (Everson et al., 2019; Liu et al., 2017; Wang et al., 2017; Yan et al., 2020; Yang et al., 2019). Hypertension, which is a main risk factor for stroke, heart disease, and renal failure(Stanaway et al., 2018), has caused 10.8 million deaths, accounting for $9.3 \%$ of disability-adjusted life years lost globally (IHME, 2019). Meanwhile, a systolic blood pressure (SBP) of at least $110 \mathrm{mmHg}$ is associated with a variety of cardiovascular and renal diseases, including ischemic heart disease, cerebrovascular disease, and chronic kidney disease (Jackson T. Wright, 2002; R Collins, 1990). Further, a positive association between diastolic blood pressure (DBP), pulse pressure (PP), and mean arterial pressure (MAP) and the risk of cardiovascular disease is common in older individuals (Chobanian et al., 2003; Howard D. Sesso, 2000). 
Therefore, it is necessary to explore the impact of indoor air pollution caused by solid fuel use on BP and hypertension.

In recent years, several studies have investigated the relationship between solid fuel use and BP and hypertension (Arku et al., 2018; Arku et al., 2020; Fatmi et al., 2019; Khan et al., 2021). However, the entire population was considered in these studies, including women, chefs, and patients with chronic diseases. One study examined 137809 individuals aged 30-70 years from 640 urban and 21 rural areas found that exposure to solid fuel pollution was negatively associated with BP and hypertension (Arku et al., 2020), but the average age of the study population was only 56 years. Three cross-section studies have evaluated the relationship between women using solid fuels and BP and hypertension, but they neglected the impact on men and had small sample sizes. A study of 77605 premenopausal women aged 15-49 years from 10 countries (Arku et al., 2018) and another study of 6543 women aged $>18$ years conducted in Bangladesh demonstrated that women using solid fuels were more likely to suffer from hypertension (Khan et al., 2021). Conversely, a study of 850 women aged $>40$ years conducted in Pakistan found no association between hypertension and indoor solid fuel use (Fatmi et al., 2019). Overall, there is limited research concerning the use of solid fuels by the elderly (aged greater than 65 years), which is the population most at risk of raised BP, had an effect on BP and hypertension (Deng et al., 2020; Lin et al., 2021).

Currently, China's average age is on the upward trend, wherein the elderly population has reached 190.64 million, accounting for $13.50 \%$ of the total population (National Bureau of Statistics of China, 2021). To date, approximately 450 million Chinese still use solid fuels for cooking (Yu et al., 2018). Thus, it is necessary to explore the relationship between indoor solid fuel use and BP in the elderly population (Gall et al., 2013; Ji, 2018). In this study, we explored the effect of using solid fuel for cooking on BP and hypertension using data from the Chinese Longitudinal Healthy Longevity Survey (CLHLS) by conducting a large population-based study including approximately 16000 individuals aged $\geq 65$ years in 500 urban and rural communities in 23 provinces in China.

\section{Methods}

\section{Study population}

In this study, we used secondary data from the 2018 CLHLS, which was a longitudinal population-based study of people over 65 years old in China. We used this data because it is the most recent available, and therefore best fits China's current population situation. Using a multi-stage stratified proportional probability sampling design, approximately 16000 elderly people in urban and rural communities were randomly selected from 500 sample areas in 23 provinces. The biomedical ethics committee of Peking University approved the study, and all study participants signed an informed consent form. In total, 8067 individuals were found eligible for this study, excluding 95 participants younger than 65 years old, 117 participants that answered "never cook", 456 participants that refused to answer, 1095 participants that answered "not applicable", 5990 participants with missing data. 


\section{BP measurement}

After a study participant rested for at least $5 \mathrm{~min}$, a research assistant measured the BP of the right arm twice using a mercury sphygmomanometer. For bedridden participants, BP was measured in the lying position. The interval between the two measurements was at least 1 min. For the SBP and DBP, we took the mean of the two measurements.

We defined hypertension patients as those with a self-reported history of hypertension, an average $\mathrm{SBP} \geq 140 \mathrm{mmHg}$ or an average DBP $\geq 90 \mathrm{~mm} \mathrm{Hg}$ (World Health Organization, 2013). Meanwhile, we defined PP as SBP-DBP and mean arterial pressure (MAP) as PP/3 + DBP to explore the possible mechanism affecting BP(Howard D. Sesso, 2000). SBP, DBP, PP, and MAP were all defined as continuous variables, whereas the prevalence of hypertension was a binary variable.

\subsection{Indoor solid fuel use assessment}

We determined the solid fuel used for cooking by asking the question, "What is the main source of cooking fuel in your family?" Participants who answered "other" were excluded from this research. Overall, we divided participants into two groups: (1) indoor solid fuel use, including coal, charcoal, and wood; and (2) clean fuel use, which includes solar energy, natural gas, induction cooker, and other electrical appliances.

\section{Covariates}

We adjusted for numerous covariates according to previous studies (Arku et al., 2020; Hystad et al., 2019; Yan et al., 2016; Yao et al., 2021). Specifically, we adjusted for demographic characteristics, including age (years), gender (female/male), marital status (Married: married and living together/Not married: widowed, divorced, separated, or never married), residence (urban/rural); socioeconomic status, including education and family income; and cardiovascular disease (CVD) risk factors, including smoking status (no/yes, participants answered "smoking now" or "in the past" was recognized as yes, as were alcohol use and exercise regularly), alcohol use (no/yes), regular exercise (no/yes), body mass index $\left(\mathrm{BMl} ;<24 \mathrm{~kg} / \mathrm{m}^{2} / \geq 24 \mathrm{~kg} / \mathrm{m}^{2}\right.$ ) (Chittaranjan S Yajnik, 2004), dietary diversity index (DDl; high: 89/median: 6-7/low: < 5) (Gina K, 2010), and use of BP lowering medication (no/yes). All self-reported information was collected by well-trained researchers via in-person family interviews.

\section{Statistical analyses}

The relationship between solid fuel for cooking and BP/hypertension was assessed using a multivariable regression model. The first crude model was unadjusted. Then we specified a base model (Model 1) that included age, gender, urban/rural status, and marital status, after which groups of CVD risk factors were progressively added. Model 2 adjusted for additional risk factors, including smoking status, alcohol use, physical activity, BMI, dietary patterns, and use of BP lowering medication. The fully adjusted 
model (Model 3) included Model 2 covariates as well as socioeconomic status, including years of education and family income.

Subgroup analyses for the association of household solid cooking fuel with hypertension prevalence or $\mathrm{BP}$ were performed according to demographic characteristics and CVD risk factors. Demographic characteristics included region (south/north, in which the provinces of southern China included Shanghai, Jiangsu, Zhejiang, Anhui, Fujian, Jiangxi, Hubei, Hunan, Guangdong, Guangxi, Hainan, Chongqing, and Sichuan; and those of northern China included Beijing, Tianjin, Hebei, Shanxi, Liaoning, Jilin, Heilongjiang, Shandong, Henan, and Shaanxi), residence (urban/rural), gender, age (elderly age/senile age/long-livers; for which the age of participants was categorized into three groups according to the age standards revised by the World Health Organization in 2015: elderly age is 60-75 years, senile age is $75-90$ years, and long-livers more than 90 years (Prusinowska et al., 2016)), indoor ventilation (none/natural/mechanical, for which participants answering "no ventilation measures" were considered as none, "naturally open windows for ventilation" were considered natural, and "range hood" and "exhaust fan" were classified as mechanical). Meanwhile, CVD risk factors included BMI $\left(<24 \mathrm{~kg} / \mathrm{m}^{2} / \geq\right.$ $24 \mathrm{~kg} / \mathrm{m}^{2}$ ), hypertension (no/yes), anti-hypertension medication use (no/yes), diabetes mellitus (no/yes), and heart diseases (no/yes).

\section{Results}

\section{Basic participant characteristics}

The characteristics of the study participants according to their indoor solid fuel cooking use are summarized in Table 1. Participants from south and north China made up $68.32 \%$ and $31.68 \%$ of the total study population, respectively. The mean age of all participants was 84.37 years. In urban areas, $21.47 \%$ of participants used indoor solid fuel for cooking, whereas in rural areas this proportion reached 41.53\%. Participants that used solid fuel had both higher SBP and DBP (urban $=138.62 \mathrm{mmHg} / 80.19$ $\mathrm{mmHg}$, rural $=141.64 \mathrm{mmHg} / 80.62 \mathrm{mmHg}$ ) than those that used clean fuel (urban $=136.58 \mathrm{mmHg}$ $/ 78.27 \mathrm{mmHg}$, rural $=139.98 \mathrm{mmHg} / 79.77 \mathrm{mmHg}$ ), showing that those who use solid fuel for cooking are at a higher risk of elevated BP. Moreover, participants who use clean fuel had higher socioeconomic indicators, both in terms of years of education and family income, than those using indoor solid fuel. 
Table 1

Characteristics of 8067 individuals with solid fuel use for cooking, stratified by urban/rural status and solid fuel use for cooking versus clean fuel.

\begin{tabular}{|c|c|c|c|c|c|}
\hline \multirow[t]{2}{*}{ Characteristic } & \multirow{2}{*}{$\begin{array}{l}\text { All } \\
\text { participants }\end{array}$} & \multicolumn{2}{|c|}{ Urban $(n=4523)$} & \multicolumn{2}{|l|}{ Rural (n=3544) } \\
\hline & & Solid fuel & Clean fuel & Solid fuel & Clean fuel \\
\hline Individuals (n, \%) & 8067 & $971(21.47)$ & $3,552(78.53)$ & $1,472(41.53)$ & $2,072(58.47)$ \\
\hline $\begin{array}{l}\mathrm{SBP}(\mathrm{mmHg}) \\
(\mathrm{mean}, \mathrm{SD})\end{array}$ & $\begin{array}{l}138.62 \\
(20.79)\end{array}$ & $\begin{array}{l}138.62 \\
(21.46)\end{array}$ & $\begin{array}{l}136.58 \\
(19.61)\end{array}$ & $\begin{array}{l}141.64 \\
(22.08)\end{array}$ & $\begin{array}{l}139.98 \\
(21.14)\end{array}$ \\
\hline $\begin{array}{l}\mathrm{DBP}(\mathrm{mmHg}) \\
\text { (mean, SD) }\end{array}$ & $\begin{array}{l}79.32 \\
(11.74)\end{array}$ & $\begin{array}{l}80.19 \\
(11.53)\end{array}$ & $\begin{array}{l}78.27 \\
(11.64)\end{array}$ & $\begin{array}{l}80.62 \\
(12.36)\end{array}$ & $\begin{array}{l}79.77 \\
(11.38)\end{array}$ \\
\hline $\begin{array}{l}\mathrm{PP}(\mathrm{mmHg}) \\
(\text { mean, SD) }\end{array}$ & $\begin{array}{l}59.30 \\
(18.08)\end{array}$ & $\begin{array}{l}58.43 \\
(18.26)\end{array}$ & $\begin{array}{l}58.30 \\
(17.91)\end{array}$ & $\begin{array}{l}61.02 \\
(18.21)\end{array}$ & $\begin{array}{l}60.21 \\
(18.10)\end{array}$ \\
\hline $\begin{array}{l}\text { MAP }(\mathrm{mmHg}) \\
(\text { mean, SD) }\end{array}$ & $\begin{array}{l}99.09 \\
(12.78)\end{array}$ & $\begin{array}{l}99.66 \\
(12.97)\end{array}$ & $\begin{array}{l}97.71 \\
(12.14)\end{array}$ & $\begin{array}{l}100.96 \\
(13.81)\end{array}$ & $\begin{array}{l}99.84 \\
(12.75)\end{array}$ \\
\hline \multicolumn{6}{|l|}{$\begin{array}{l}\text { Hypertension (n, } \\
\% \text { ) }\end{array}$} \\
\hline No & $\begin{array}{l}2,998 \\
(37.16)\end{array}$ & $410(42.22)$ & $1,283(36.12)$ & $563(38.25)$ & $742(35.81)$ \\
\hline Yes & $\begin{array}{l}5,069 \\
(62.84)\end{array}$ & $561(57.78)$ & $2,269(63.88)$ & 909 (61.75) & $1,330(64.1)$ \\
\hline \multicolumn{6}{|l|}{ Region (n, \%) } \\
\hline South & $\begin{array}{l}5,511 \\
(68.32)\end{array}$ & $665(68.49)$ & $2,348(66.10)$ & 932 (63.32) & $1,566(75.58)$ \\
\hline North & $\begin{array}{l}2,556 \\
(31.68)\end{array}$ & $306(31.51)$ & $1,204(33.90)$ & $540(36.68)$ & $506(24.42)$ \\
\hline $\begin{array}{l}\text { Age (years, mean, } \\
\text { SD) }\end{array}$ & $\begin{array}{l}84.37 \\
(11.82)\end{array}$ & $\begin{array}{l}84.28 \\
(11.83)\end{array}$ & $\begin{array}{l}84.55 \\
(11.78)\end{array}$ & $\begin{array}{l}84.27 \\
(11.76)\end{array}$ & $\begin{array}{l}84.18 \\
(11.95)\end{array}$ \\
\hline \multicolumn{6}{|l|}{$\operatorname{Sex}(n, \%)$} \\
\hline Female & $\begin{array}{l}4,457 \\
(55.25)\end{array}$ & $540(55.61)$ & 1,888 (53.15) & $859(58.36)$ & $1,170(56.47)$ \\
\hline Male & $\begin{array}{l}3,610 \\
(44.75)\end{array}$ & $431(44.39)$ & $1,664(46.85)$ & $613(41.64)$ & $902(43.53)$ \\
\hline $\begin{array}{l}\text { Marital status (n, } \\
\%)\end{array}$ & & & & & \\
\hline
\end{tabular}

a Dietary diversity: Low (Dietary Diversity Index [DDI] $\leq 4$ ); median (DDI: 5-6); high (DDI: $\geq 7$ ).

Abbreviation: SBP, systolic blood pressure; DBP, diastolic blood pressure; BMI, body mass index; SD, standard deviation. 


\begin{tabular}{|c|c|c|c|c|c|}
\hline \multirow[t]{2}{*}{ Characteristic } & \multirow{2}{*}{$\begin{array}{l}\text { All } \\
\text { participants }\end{array}$} & \multicolumn{2}{|c|}{ Urban $(n=4523)$} & \multicolumn{2}{|l|}{ Rural (n=3544) } \\
\hline & & Solid fuel & Clean fuel & Solid fuel & Clean fuel \\
\hline Not Married & $\begin{array}{l}4,446 \\
(55.11)\end{array}$ & $515(53.04)$ & $1,950(54.90)$ & 795 (54.01) & $1,186(57.24)$ \\
\hline Married & $\begin{array}{l}3,621 \\
(44.89)\end{array}$ & $456(46.96)$ & $1,602(45.10)$ & 677 (45.99) & $886(42.76)$ \\
\hline $\begin{array}{l}\text { Education (years, } \\
\text { mean, SD) }\end{array}$ & $3.33(4.13)$ & $2.00(2.95)$ & 4.59 (4.79) & $2.09(2.94)$ & $2.68(3.46)$ \\
\hline $\begin{array}{l}\text { Income (yuan, } \\
\text { mean, SD) }\end{array}$ & $\begin{array}{l}41687.28 \\
(36244.09)\end{array}$ & $\begin{array}{l}24439.89 \\
(28362.57)\end{array}$ & $\begin{array}{l}56120.33 \\
(35899.06)\end{array}$ & $\begin{array}{l}22010.39 \\
(26988.49)\end{array}$ & $\begin{array}{l}39006.51 \\
(35118.00)\end{array}$ \\
\hline \multicolumn{6}{|l|}{$\begin{array}{l}\text { Smoking status } \\
(n, \%)\end{array}$} \\
\hline No & $\begin{array}{l}5,537 \\
(68.64)\end{array}$ & $665(68.49)$ & 2,427 (68.33) & 1,045 (70.99) & $1,400(67.57)$ \\
\hline Yes & $\begin{array}{l}2,530 \\
(31.36)\end{array}$ & $306(31.51)$ & $1,125(31.67)$ & $427(29.01)$ & $672(32.43)$ \\
\hline \multicolumn{6}{|l|}{ Alcohol use (n, \%) } \\
\hline No & $\begin{array}{l}5,879 \\
(72.88)\end{array}$ & $682(70.24)$ & 2,609 (73.45) & $1,100(74.73)$ & $1,488(71.81)$ \\
\hline Yes & $\begin{array}{l}2,188 \\
(27.12)\end{array}$ & $289(29.76)$ & $943(26.55)$ & $372(25.27)$ & $584(28.19)$ \\
\hline \multicolumn{6}{|l|}{$\begin{array}{l}\text { Exercise regularly } \\
(\mathrm{n}, \%)\end{array}$} \\
\hline No & $\begin{array}{l}4,750 \\
(58.88)\end{array}$ & $722(74.36)$ & $1,563(44.00)$ & $1,091(74.12)$ & $1,374(66.31)$ \\
\hline Yes & $\begin{array}{l}3,317 \\
(41.12)\end{array}$ & $249(25.64)$ & $1,989(56.00)$ & $381(25.88)$ & 698 (33.69) \\
\hline \multicolumn{6}{|l|}{ BMI (n, \%) } \\
\hline$<24$ & $\begin{array}{l}5,480 \\
(67.93)\end{array}$ & $724(74.56)$ & $2,267(63.82)$ & $1,074(72.96)$ & 1,415 (68.29) \\
\hline$\geq 24$ & $\begin{array}{l}2,587 \\
(32.07)\end{array}$ & $247(25.44)$ & $1,285(36.18)$ & $398(27.04)$ & 657 (31.71) \\
\hline \multicolumn{6}{|l|}{$\begin{array}{l}\text { Dietary diversity }{ }^{a} \\
(n, \%)\end{array}$} \\
\hline \multicolumn{6}{|c|}{ a Dietary diversity: Low (Dietary Diversity Index [DDI] $\leq 4)$; median (DDI: 5-6); high (DDI: $\geq 7$ ). } \\
\hline $\begin{array}{l}\text { Abbreviation: SBP, } \\
\text { standard deviation }\end{array}$ & is & e; DBP, dic & blood pres & BMI, body $r$ & index; SD, \\
\hline
\end{tabular}




\begin{tabular}{|c|c|c|c|c|c|}
\hline \multirow[t]{2}{*}{ Characteristic } & \multirow{2}{*}{$\begin{array}{l}\text { All } \\
\text { participants }\end{array}$} & \multicolumn{2}{|c|}{ Urban $(n=4523)$} & \multicolumn{2}{|l|}{ Rural ( $n=3544)$} \\
\hline & & Solid fuel & Clean fuel & Solid fuel & Clean fuel \\
\hline Low & $\begin{array}{l}1,425 \\
(17.66)\end{array}$ & 264 (27.19) & $416(11.71)$ & 354 (24.05) & 391 (18.87) \\
\hline Median & $\begin{array}{l}3,185 \\
(39.48)\end{array}$ & 444 (45.73) & $1,190(33.50)$ & 645 (43.82) & 906 (43.73) \\
\hline High & $\begin{array}{l}3,457 \\
(42.85)\end{array}$ & $263(27.09)$ & $1,946(54.79)$ & $473(32.13)$ & $775(37.40)$ \\
\hline \multicolumn{6}{|c|}{$\begin{array}{l}\text { Anti-hypertension medication }(\mathrm{n}, \\
\%)\end{array}$} \\
\hline No & $\begin{array}{l}4,848 \\
(60.10)\end{array}$ & $638(65.71)$ & $1,980(55.74)$ & $978(66.44)$ & $1,252(60.42)$ \\
\hline Yes & $\begin{array}{l}3,219 \\
(39.90)\end{array}$ & 333 (34.29) & $1,572(44.26)$ & $494(33.56)$ & 820 (39.58) \\
\hline \multicolumn{6}{|c|}{$\begin{array}{l}\text { Indoor ventilation } \\
(\mathrm{n}, \%)\end{array}$} \\
\hline None & $696(8.66)$ & $151(15.66)$ & 177 (4.99) & $200(13.73)$ & $168(8.14)$ \\
\hline Natural & $\begin{array}{l}3,784 \\
(47.11)\end{array}$ & 715 (74.17) & $828(23.34)$ & $1,147(78.72)$ & $1,094(52.98)$ \\
\hline Mechanical & $\begin{array}{l}3,553 \\
(44.23)\end{array}$ & 98 (10.17) & $2,542(71.67)$ & $110(7.55)$ & 803 (38.89) \\
\hline \multicolumn{6}{|l|}{ Diabetes (n, \%) } \\
\hline No & $\begin{array}{l}6,639 \\
(85.38)\end{array}$ & 824 (88.70) & 2,806 (82.05) & $1,285(90.43)$ & 1,724 (85.94) \\
\hline Yes & $\begin{array}{l}1,137 \\
(14.62)\end{array}$ & $105(11.30)$ & 614 (17.95) & $136(9.57)$ & $282(14.06)$ \\
\hline \multicolumn{6}{|c|}{$\begin{array}{l}\text { Heart disease }(\mathrm{n}, \\
\%)\end{array}$} \\
\hline No & $\begin{array}{l}6,194 \\
(79.69)\end{array}$ & 782 (84.09) & $2,601(76.10)$ & $1,193(83.95)$ & $1,618(80.74)$ \\
\hline Yes & $\begin{array}{l}1,579 \\
(20.31)\end{array}$ & 148 (15.91) & $817(23.90)$ & 228 (16.05) & 386 (19.26) \\
\hline \multicolumn{6}{|c|}{ a Dietary diversity: Low (Dietary Diversity Index [DDI] $\leq 4)$; median (DDI: 5-6); high (DDI: $\geq 7$ ). } \\
\hline $\begin{array}{l}\text { Abbreviation: } \\
\text { standard devi }\end{array}$ & lic blood & Ire; DBP, dic & blood pres & BMI, body $n$ & index; SD, \\
\hline
\end{tabular}




\section{Distribution Of Indoor Solid Fuel Use Across Provinces}

Figure 1 shows the location of the study communities, the proportion of cooking with indoor solid fuel in each province (Fig. 1A), and the per capita distribution of resident disposable income in an interquartile range (Fig. 1B). As shown in Fig. $1 \mathrm{~A}$, less than $10 \%$ of the population residing in the provinces of Beijing, Fujian, Guangdong, Hebei, Heilongjiang, Shanghai, Tianjin, and Zhejiang used solid fuel, whereas in Anhui, Hunan, Jilin, Jiangxi, and Shandong, nearly $50 \%$ of the population used indoor solid fuel. As shown in Fig. 1B, low-income and lower middle-income provinces had the highest solid fuel exposure, wherein the proportion of people using solid fuel reached $36 \%$. Conversely, high-income provinces had the lowest proportion of cooking with indoor solid fuel, at only $17 \%$.

\section{Association of indoor solid fuel use with BP and hypertension}

Table 2 summarizes the association between indoor solid fuel use and BP. The unadjusted Model and Model 1 revealed that solid fuel use for cooking is strongly associated with increased BP. Model 1 also showed that people who cooked primarily with indoor solid fuel had a lower likelihood of hypertension $(\mathrm{OR}=0.83 ; 95 \% \mathrm{Cl}: 0.75,0.92)$. Model 2 , which included CVD risk factors, produced the same results as Model 1, but showed an increased effect of solid fuel on BP. Meanwhile, Model 3, which included socioeconomic status measures, revealed weaker association between solid fuel use and $\mathrm{BP} /$ hypertension. Overall, compared with those who cooked with clean fuel, those who cooked with solid fuel had a $1.87 \mathrm{mmHg}$ higher SBP (95\% Cl: 0.85, 2.89), a $0.09 \mathrm{mmHg}$ higher DBP (95\% Cl: 0.31, 1.49), a $0.97 \mathrm{mmHg}$ higher PP (95\% Cl: $0.06,1.88)$, and a $1.22 \mathrm{mmHg}$ higher MAP ( $95 \% \mathrm{Cl}: 0.59,1.85)$. However, there was no significant association observed with hypertension ( $\mathrm{OR}=0.99,95 \% \mathrm{Cl}: 0.87,1.14)$. 
Table 2

Associations between use of solid fuels for cooking, versus clean fuels, and BP parameters and the odds of having hypertension.

\begin{tabular}{|c|c|c|c|c|c|}
\hline & SBP & DBP & PP & MAP & Hypertension \\
\hline & $\beta(95 \% \mathrm{Cl})$ & $\beta(95 \% \mathrm{Cl})$ & $\beta(95 \% \mathrm{Cl})$ & $\beta(95 \% \mathrm{Cl})$ & OR (95\% Cl) \\
\hline $\begin{array}{l}\text { Crude } \\
\text { model }\end{array}$ & $\begin{array}{l}2.60(1.62 \\
3.59) * \star \star\end{array}$ & $\begin{array}{l}1.62(1.06 \\
2.18) * \star * \star\end{array}$ & $\begin{array}{l}0.99(0.13 \\
1.85) *\end{array}$ & $\begin{array}{l}1.95(1.34 \\
2.55) * \star \star\end{array}$ & $\begin{array}{l}0.85(0.77 \\
0.94) \star \star\end{array}$ \\
\hline Model 1 & $\begin{array}{l}1.82(0.81 \\
2.82) \star \star \star\end{array}$ & $\begin{array}{l}1.35(0.79 \\
1.92) \star \star \star\end{array}$ & $\begin{array}{l}0.46(-0.41 \\
1.34)\end{array}$ & $\begin{array}{l}1.51(0.89 \\
2.13) \star \star \star\end{array}$ & $\begin{array}{l}0.83(0.75 \\
0.92) \star \star \star\end{array}$ \\
\hline Model 2 & $\begin{array}{l}2.72(1.73 \\
3.70) \star \star \star\end{array}$ & $\begin{array}{l}1.63(1.06 \\
2.20)^{\star \star \star}\end{array}$ & $\begin{array}{l}1.09(0.21 \\
1.96)^{\star}\end{array}$ & $\begin{array}{l}1.99(1.38 \\
2.60) \star \star \star\end{array}$ & $\begin{array}{l}1.05(0.92 \\
1.19)\end{array}$ \\
\hline Model 3 & $\begin{array}{l}1.87(0.85 \\
2.89) \star * \star\end{array}$ & $\begin{array}{l}0.90(0.31 \\
1.49) * *\end{array}$ & $\begin{array}{l}0.97(0.06 \\
1.88)^{*}\end{array}$ & $\begin{array}{l}1.22(0.59 \\
1.85) \star * *\end{array}$ & $\begin{array}{l}0.99(0.87 \\
1.14)\end{array}$ \\
\hline \multicolumn{6}{|c|}{$\begin{array}{l}\text { Note: Model 1: age, gender, urban/rural status, marital status; Model 2: Model 1+ smoking status, } \\
\text { alcohol use, physical activity, BMI, dietary diversity, use of BP lowering medication; Model 3: Model 2+ } \\
\text { education, income. }\end{array}$} \\
\hline \multicolumn{6}{|c|}{ 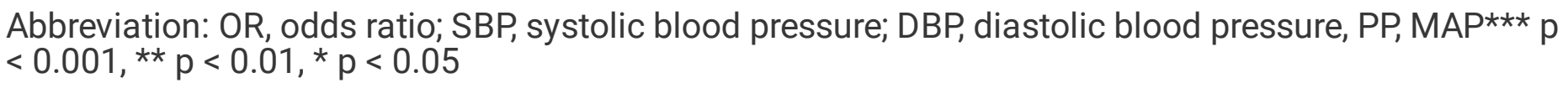 } \\
\hline
\end{tabular}

\section{Subgroup analyses of indoor solid fuel use with BP and hypertension}

Table 3 lists the results of the stratified analysis and interaction effect. Regarding geographic location, we found that the SBP of residents using solid fuel in north China $(95 \% \mathrm{Cl}: 0.13,1.64)$ was $0.25 \mathrm{mmHg}$ higher than that of residents in south China $(95 \% \mathrm{Cl}: 0.00,2.28)$, for which the interaction term was significant $(p<0.001)$. Meanwhile, in terms of age, the results showed that long-livers who cooked with solid fuel had a SBP $3.37 \mathrm{mmHg}(95 \% \mathrm{Cl}: 1.57,5.17)$ higher than those who cooked with clean fuel, and that there was a significant interaction term $(p<0.001)$. It was found that for women aged over 65 years, using solid fuel greatly impacted $\mathrm{BP}(\beta=2.76 \mathrm{mmHg}, 95 \% \mathrm{Cl}$ : $1.32,4.19$ for SBP; $\beta=1.08 \mathrm{mmHg}, 95 \% \mathrm{Cl}$ : $0.27,1.89)$. In addition, heart disease patients using indoor solid fuel had a $1.57 \mathrm{mmHg}$ higher DBP than those who cooked with clean fuel $(95 \% \mathrm{Cl}: 0.10,3.04)$. Regarding indoor ventilation, we observed a strong association between indoor solid fuel use for cooking and increased SBP/DBP. Specifically, if a kitchen was naturally ventilated when cooking, people who cooked with solid fuel had an SBP $1.81 \mathrm{mmHg}(95 \%$ Cl: $0.47,3.15)$ and a DBP $1.18 \mathrm{mmHg}(\mathrm{Cl}: 0.43,1.93)$ higher than those who cooked with clean fuel. Nevertheless, we did not find that using indoor solid fuel was related to BP or hypertension in the case of those using mechanical ventilation and those with no ventilation. 
Table 3

Model results for multivariable analysis of the association of BP parameters and odds of hypertension with solid fuel use, stratified by selected variables

\begin{tabular}{|c|c|c|c|c|c|c|}
\hline \multirow[t]{2}{*}{$\begin{array}{l}\text { Entire } \\
\text { sample }\end{array}$} & $\mathbf{N}$ & $\begin{array}{l}\text { Systolic BP } \\
\beta(95 \% \text { Cl) }\end{array}$ & $\begin{array}{l}\text { Diastolic } \\
\text { BP } \\
\beta(95 \% \mathrm{Cl})\end{array}$ & $\begin{array}{l}\mathrm{PP} \\
\beta(95 \% \mathrm{Cl})\end{array}$ & $\begin{array}{l}\text { MAP } \\
\beta(95 \% \mathrm{Cl})\end{array}$ & $\begin{array}{l}\text { Hypertension } \\
\text { OR }(95 \% \mathrm{Cl})\end{array}$ \\
\hline & 8067 & $\begin{array}{l}1.87(0.85, \\
2.89)\end{array}$ & $\begin{array}{l}0.90(0.31, \\
1.49)\end{array}$ & $\begin{array}{l}0.97(0.06, \\
1.88)\end{array}$ & $\begin{array}{l}1.22(0.59, \\
1.85)\end{array}$ & $\begin{array}{l}0.99(0.87, \\
1.14)\end{array}$ \\
\hline \multicolumn{7}{|l|}{ Region } \\
\hline South & 5511 & $\begin{array}{l}0.67(-0.56 \\
1.89)\end{array}$ & $\begin{array}{l}1.00(0.31 \\
1.70)\end{array}$ & $\begin{array}{l}-0.33(-1.39 \\
0.73)\end{array}$ & $\begin{array}{l}0.89 \\
(0.13,1.64) \\
\star\end{array}$ & $\begin{array}{l}0.91(0.77, \\
1.07)\end{array}$ \\
\hline North & 2556 & $\begin{array}{l}3.50(1.65 \\
5.35) \star \star \star\end{array}$ & $\begin{array}{l}-0.03 \\
(-1.16,1.08)\end{array}$ & $\begin{array}{l}3.54(1.79 \\
5.29)\end{array}$ & $\begin{array}{l}1.14(0.00 \\
2.28)^{*}\end{array}$ & $\begin{array}{l}1.12(0.87, \\
1.44)\end{array}$ \\
\hline $\begin{array}{l}\text { Interaction } \\
\text { effect }\end{array}$ & & $\begin{array}{l}3.40(1.88 \\
4.92) \star \star \star\end{array}$ & $\begin{array}{l}1.67(0.82 \\
2.53) \star \star \star \star\end{array}$ & $\begin{array}{l}1.72(0.39 \\
3.06) *\end{array}$ & $\begin{array}{l}2.25(1.32 \\
3.18) \star \star \star\end{array}$ & $\begin{array}{l}1.06(0.91, \\
1.25)\end{array}$ \\
\hline \multicolumn{7}{|c|}{ Community Location } \\
\hline Urban & 4523 & $\begin{array}{l}1.49(-0.00 \\
2.99)\end{array}$ & $\begin{array}{l}0.78(-0.11 \\
1.66)\end{array}$ & $\begin{array}{l}0.72(-0.65 \\
2.09) *\end{array}$ & $\begin{array}{l}1.01(0.09 \\
1.94)^{*}\end{array}$ & $\begin{array}{l}0.89(0.72 \\
1.10)\end{array}$ \\
\hline Rural & 3544 & $\begin{array}{l}2.07(0.65 \\
3.49) \star \star\end{array}$ & $\begin{array}{l}0.94(0.15 \\
1.73)^{\star}\end{array}$ & $\begin{array}{l}1.13(-0.10 \\
2.35)\end{array}$ & $\begin{array}{l}1.32(0.45 \\
2.19) \star \star\end{array}$ & $\begin{array}{l}1.06(0.89 \\
1.27)\end{array}$ \\
\hline $\begin{array}{l}\text { Interaction } \\
\text { effect }\end{array}$ & & $\begin{array}{l}1.53(-0.02 \\
3.09)\end{array}$ & $\begin{array}{l}1.14(0.26 \\
2.01)^{\star}\end{array}$ & $\begin{array}{l}0.40(-0.97 \\
1.76)\end{array}$ & $\begin{array}{l}1.27(0.32 \\
2.22) \star \star\end{array}$ & $\begin{array}{l}0.91(0.78 \\
1.06)\end{array}$ \\
\hline \multicolumn{7}{|l|}{ Gender } \\
\hline Female & 4457 & $\begin{array}{l}2.76(1.32 \\
4.19) \star \star \star\end{array}$ & $\begin{array}{l}1.08(0.27 \\
1.89) * \star\end{array}$ & $\begin{array}{l}1.68(0.37 \\
2.99) *\end{array}$ & $\begin{array}{l}1.64(0.77 \\
2.50) * \star \star\end{array}$ & $\begin{array}{l}1.00(0.84 \\
1.20)\end{array}$ \\
\hline Male & 3610 & $\begin{array}{l}0.71(-0.72, \\
2.13)\end{array}$ & $\begin{array}{l}0.68(-0.17 \\
1.54)\end{array}$ & $\begin{array}{l}0.02(-1.20 \\
1.25)\end{array}$ & $\begin{array}{l}0.69(-0.22, \\
1.60)\end{array}$ & $\begin{array}{l}0.99(0.80 \\
1.21)\end{array}$ \\
\hline $\begin{array}{l}\text { Interaction } \\
\text { effect }\end{array}$ & & $\begin{array}{l}0.16(-1.38 \\
1.71)\end{array}$ & $\begin{array}{l}0.27(-0.60 \\
1.13)\end{array}$ & $\begin{array}{l}-0.11(-1.46, \\
1.25)\end{array}$ & $\begin{array}{l}0.23(-0.71 \\
1.18)\end{array}$ & $\begin{array}{l}0.92(0.79 \\
1.08)\end{array}$ \\
\hline \multicolumn{7}{|l|}{ Age } \\
\hline Elderly age & 2056 & $\begin{array}{l}-0.40(-2.26 \\
1.46)\end{array}$ & $\begin{array}{l}0.22(-0.85 \\
1.30)\end{array}$ & $\begin{array}{l}-0.62(-2.20, \\
0.96)\end{array}$ & $\begin{array}{l}0.02(-1.16, \\
1.19)\end{array}$ & $\begin{array}{l}0.88(0.66 \\
1.16)\end{array}$ \\
\hline Senile age & 2992 & $\begin{array}{l}1.94(0.32 \\
3.56) *\end{array}$ & $\begin{array}{l}0.80(-0.20 \\
1.79)\end{array}$ & $\begin{array}{l}1.14(-0.36 \\
2.64)\end{array}$ & $\begin{array}{l}1.18(0.16 \\
2.20)^{*}\end{array}$ & $\begin{array}{l}0.86(0.68 \\
1.10)\end{array}$ \\
\hline Long-livers & 3019 & $\begin{array}{l}3.37(1.57 \\
5.17)^{\star \star \star}\end{array}$ & $\begin{array}{l}1.53(0.54 \\
2.52) * \star\end{array}$ & $\begin{array}{l}1.84(0.25 \\
3.44) \star\end{array}$ & $\begin{array}{l}2.14(1.06 \\
3.22)^{\star \star \star}\end{array}$ & $\begin{array}{l}1.15(0.94 \\
1.41)\end{array}$ \\
\hline
\end{tabular}

Abbreviation: BP, blood pressure; BMI, body mass index. ${ }^{* \star *} p<0.001,{ }^{* *} p<0.01,{ }^{*} p<0.05$ 


\begin{tabular}{|c|c|c|c|c|c|c|}
\hline \multirow[t]{2}{*}{$\begin{array}{l}\text { Entire } \\
\text { sample }\end{array}$} & $\mathbf{N}$ & $\begin{array}{l}\text { Systolic BP } \\
\beta(95 \% \mathrm{Cl})\end{array}$ & $\begin{array}{l}\text { Diastolic } \\
\text { BP } \\
\beta(95 \% \mathrm{Cl})\end{array}$ & $\begin{array}{l}\text { PP } \\
\beta(95 \% \mathrm{Cl})\end{array}$ & $\begin{array}{l}\text { MAP } \\
\beta(95 \% \mathrm{Cl})\end{array}$ & $\begin{array}{l}\text { Hypertension } \\
\text { OR }(95 \% \mathrm{Cl})\end{array}$ \\
\hline & 8067 & $\begin{array}{l}1.87(0.85 \\
2.89)\end{array}$ & $\begin{array}{l}0.90(0.31 \\
1.49)\end{array}$ & $\begin{array}{l}0.97(0.06, \\
1.88)\end{array}$ & $\begin{array}{l}1.22(0.59 \\
1.85)\end{array}$ & $\begin{array}{l}0.99(0.87 \\
1.14)\end{array}$ \\
\hline $\begin{array}{l}\text { Interaction } \\
\text { effect }\end{array}$ & & $\begin{array}{l}0.86(0.39 \\
1.32) * \star \star \star\end{array}$ & $\begin{array}{l}0.43(0.17 \\
0.69)^{\star \star}\end{array}$ & $\begin{array}{l}0.43(0.03 \\
0.84) *\end{array}$ & $\begin{array}{l}0.57(0.29 \\
0.85) * \star \star\end{array}$ & $\begin{array}{l}0.99(0.95 \\
1.04)\end{array}$ \\
\hline \multicolumn{7}{|l|}{ BMI } \\
\hline$<24\left(\mathrm{~kg} / \mathrm{m}^{2}\right)$ & 5480 & $\begin{array}{l}1.67(0.45 \\
2.90) \star \star\end{array}$ & $\begin{array}{l}0.76(0.06 \\
1.46) \star\end{array}$ & $\begin{array}{l}0.92(-0.19 \\
2.02)\end{array}$ & $\begin{array}{l}1.06(0.32 \\
1.81)^{\star \star \star}\end{array}$ & $\begin{array}{l}0.88(0.76 \\
1.04)\end{array}$ \\
\hline$\geq 24\left(\mathrm{~kg} / \mathrm{m}^{2}\right)$ & 2587 & $\begin{array}{l}2.53(0.68 \\
4.38) * \star\end{array}$ & $\begin{array}{l}1.32(0.22 \\
2.42) *\end{array}$ & $\begin{array}{l}1.21(-0.41 \\
2.83)\end{array}$ & $\begin{array}{l}1.72(0.56 \\
2.89) * \star\end{array}$ & $\begin{array}{l}1.48(1.11 \\
1.96)^{\star \star}\end{array}$ \\
\hline $\begin{array}{l}\text { Interaction } \\
\text { effect }\end{array}$ & & $\begin{array}{l}2.95(1.17 \\
4.72) \star \star\end{array}$ & $\begin{array}{l}1.98(0.98 \\
2.97) \star \star \star \star\end{array}$ & $\begin{array}{l}0.97(-0.58 \\
2.52)\end{array}$ & $\begin{array}{l}2.30(1.22 \\
3.38) \star \star \star \star\end{array}$ & $\begin{array}{l}1.28(1.05 \\
1.57)^{*}\end{array}$ \\
\hline \multicolumn{7}{|l|}{ Hypertension } \\
\hline No & 2998 & $\begin{array}{l}0.45(-0.49 \\
1.40)\end{array}$ & $\begin{array}{l}0.20(-0.49 \\
0.90)\end{array}$ & $\begin{array}{l}0.25(-0.61 \\
1.11)\end{array}$ & $\begin{array}{l}0.29(-0.38 \\
0.96)\end{array}$ & NA \\
\hline Yes & 5069 & $\begin{array}{l}2.80(1.50 \\
4.10) \star \star \star \star\end{array}$ & $\begin{array}{l}1.40(0.60 \\
2.19) \star \star\end{array}$ & $\begin{array}{l}1.41(0.15 \\
2.67) \star\end{array}$ & $\begin{array}{l}1.86(1.07 \\
2.66) \star \star \star \star\end{array}$ & NA \\
\hline $\begin{array}{l}\text { Interaction } \\
\text { effect }\end{array}$ & & $\begin{array}{l}15.08(13.92 \\
16.25) * * \star\end{array}$ & $\begin{array}{l}5.73(5.06 \\
6.40) \star \star \star \star\end{array}$ & $\begin{array}{l}9.36(8.32 \\
10.40) * \star \star\end{array}$ & $\begin{array}{l}8.85(8.14 \\
9.56) \star \star \star\end{array}$ & NA \\
\hline \multicolumn{7}{|c|}{ Anti-hypertension medication use } \\
\hline No & 4848 & $\begin{array}{l}0.64(-0.59 \\
1.87)\end{array}$ & $\begin{array}{l}0.45(-0.22 \\
1.13)\end{array}$ & $\begin{array}{l}0.19(-0.88 \\
1.26)\end{array}$ & $\begin{array}{l}0.51(-0.23 \\
1.26)\end{array}$ & $\begin{array}{l}1.00(0.87, \\
1.14)\end{array}$ \\
\hline Yes & 3219 & $\begin{array}{l}4.28(2.51 \\
6.06) * \star \star \star\end{array}$ & $\begin{array}{l}1.91(0.82 \\
3.00) \star \star \star\end{array}$ & $\begin{array}{l}2.38(0.73 \\
4.02) \star \star\end{array}$ & $\begin{array}{l}2.70(1.59 \\
3.82) * \star \star \star\end{array}$ & NA \\
\hline $\begin{array}{l}\text { Interaction } \\
\text { effect }\end{array}$ & & $\begin{array}{l}5.09(3.48 \\
6.70) \star \star \star\end{array}$ & $\begin{array}{l}2.73(1.80 \\
3.66) \star \star \star \star\end{array}$ & $\begin{array}{l}2.36(0.93 \\
3.80) \star \star\end{array}$ & $\begin{array}{l}3.52(2.53 \\
4.50) * \star \star\end{array}$ & NA \\
\hline \multicolumn{7}{|c|}{ Indoor ventilation } \\
\hline None & 696 & $\begin{array}{l}0.01(-3.01 \\
3.03)\end{array}$ & $\begin{array}{l}-0.17(-1.87, \\
1.53)\end{array}$ & $\begin{array}{l}0.18(-2.44 \\
2.80)\end{array}$ & $\begin{array}{l}-0.11(-1.97 \\
1.75)\end{array}$ & $\begin{array}{l}1.26(0.83 \\
1.90)\end{array}$ \\
\hline Natural & 3784 & $\begin{array}{l}1.81(0.47 \\
3.15)^{\star \star}\end{array}$ & $\begin{array}{l}1.18(0.43 \\
1.93) \star \star\end{array}$ & $\begin{array}{l}0.63(-0.54 \\
1.79)\end{array}$ & $\begin{array}{l}1.39(0.57 \\
2.21)^{\star \star}\end{array}$ & $\begin{array}{l}0.95(0.80 \\
1.13)\end{array}$ \\
\hline Mechanical & 3553 & $\begin{array}{l}0.04(-2.65 \\
2.74)\end{array}$ & $\begin{array}{l}-0.67(-2.30, \\
0.95)\end{array}$ & $\begin{array}{l}0.72(-1.78 \\
3.22)\end{array}$ & $\begin{array}{l}-0.43(-2.11, \\
1.24)\end{array}$ & $\begin{array}{l}0.72(0.49, \\
1.07)\end{array}$ \\
\hline
\end{tabular}

Abbreviation: BP, blood pressure; BMI, body mass index. ${ }^{* * *} p<0.001,{ }^{* *} p<0.01,{ }^{*} p<0.05$ 


\begin{tabular}{|c|c|c|c|c|c|c|}
\hline \multirow[t]{2}{*}{$\begin{array}{l}\text { Entire } \\
\text { sample }\end{array}$} & $\mathbf{N}$ & $\begin{array}{l}\text { Systolic BP } \\
\beta(95 \% \mathrm{Cl})\end{array}$ & $\begin{array}{l}\text { Diastolic } \\
\text { BP } \\
\beta(95 \% \mathrm{Cl})\end{array}$ & $\begin{array}{l}\text { PP } \\
\beta(95 \% \mathrm{Cl})\end{array}$ & $\begin{array}{l}\text { MAP } \\
\beta(95 \% \mathrm{Cl})\end{array}$ & $\begin{array}{l}\text { Hypertension } \\
\text { OR }(95 \% \mathrm{Cl})\end{array}$ \\
\hline & 8067 & $\begin{array}{l}1.87(0.85 \\
2.89)\end{array}$ & $\begin{array}{l}0.90(0.31 \\
1.49)\end{array}$ & $\begin{array}{l}0.97(0.06, \\
1.88)\end{array}$ & $\begin{array}{l}1.22(0.59 \\
1.85)\end{array}$ & $\begin{array}{l}0.99(0.87, \\
1.14)\end{array}$ \\
\hline $\begin{array}{l}\text { Interaction } \\
\text { effect }\end{array}$ & & $\begin{array}{l}0.61(0.08 \\
1.13) \star\end{array}$ & $\begin{array}{l}0.28(-0.01 \\
0.59)\end{array}$ & $\begin{array}{l}0.32(-0.14 \\
0.78)\end{array}$ & $\begin{array}{l}0.39(0.07 \\
0.71)^{\star}\end{array}$ & $\begin{array}{l}0.95(0.90 \\
1.01)\end{array}$ \\
\hline \multicolumn{7}{|c|}{ Diabetes mellitus } \\
\hline No & 6639 & $\begin{array}{l}1.96(0.84 \\
3.07) \star \star\end{array}$ & $\begin{array}{l}0.87(0.23 \\
1.50) \star \star\end{array}$ & $\begin{array}{l}1.09(0.10 \\
2.08)^{\star}\end{array}$ & $\begin{array}{l}1.23(0.55 \\
1.91) \star \star \star\end{array}$ & $\begin{array}{l}0.99(0.86 \\
1.14)\end{array}$ \\
\hline Yes & 1137 & $\begin{array}{l}0.25(-2.68 \\
3.25)\end{array}$ & $\begin{array}{l}1.17(-0.61 \\
2.95)\end{array}$ & $\begin{array}{l}-0.88(-3.63 \\
1.87)\end{array}$ & $\begin{array}{l}0.87(-0.96 \\
2.70)\end{array}$ & $\begin{array}{l}1.70(0.93 \\
3.08)\end{array}$ \\
\hline $\begin{array}{l}\text { Interaction } \\
\text { effect }\end{array}$ & & $\begin{array}{l}0.37(-2.58 \\
3.32)\end{array}$ & $\begin{array}{l}2.01(0.36 \\
3.66)^{\star}\end{array}$ & $\begin{array}{l}-1.64(-4.23, \\
0.95)\end{array}$ & $\begin{array}{l}1.46(-0.33 \\
3.26)\end{array}$ & $\begin{array}{l}1.76(1.08 \\
2.88) \star\end{array}$ \\
\hline \multicolumn{7}{|l|}{$\begin{array}{l}\text { Heart } \\
\text { disease }\end{array}$} \\
\hline No & 6194 & $\begin{array}{l}1.57(0.42 \\
2.72) \star \star\end{array}$ & $\begin{array}{l}0.71(0.06 \\
1.36)^{\star}\end{array}$ & $\begin{array}{l}0.86(-0.16 \\
1.89)\end{array}$ & $\begin{array}{l}1.00(0.30 \\
1.70)^{\star \star}\end{array}$ & $\begin{array}{l}0.97(0.84 \\
1.13)\end{array}$ \\
\hline Yes & 1579 & $\begin{array}{l}2.29(-0.15 \\
4.74)\end{array}$ & $\begin{array}{l}1.57(0.10 \\
3.04) \star\end{array}$ & $\begin{array}{l}0.72(-1.52 \\
2.97)\end{array}$ & $\begin{array}{l}1.81(0.29 \\
3.34)^{\star}\end{array}$ & $\begin{array}{l}1.12(0.78 \\
1.61)\end{array}$ \\
\hline $\begin{array}{l}\text { Interaction } \\
\text { effect }\end{array}$ & & $\begin{array}{l}1.70(-0.71 \\
4.12)\end{array}$ & $\begin{array}{l}1.73(0.38 \\
3.08) \star\end{array}$ & $\begin{array}{l}-0.03(-2.15 \\
2.10)\end{array}$ & $\begin{array}{l}1.72(0.25 \\
3.19) *\end{array}$ & $\begin{array}{l}0.94(0.71 \\
1.25)\end{array}$ \\
\hline
\end{tabular}

\section{Meta-analysis Of Regional Impact On Indoor Air Pollution}

Meta-analysis of regional impact on indoor air pollution

Finally, the association between solid fuel use for cooking and BP was explored using a meta-analysis according to geographic location. As shown in Fig. 2, the overall pooled $\beta$ for SBP was 1.57 (95 Cl\%: 0.49, 2.64) with $\mathrm{I}^{2}=0$ and $\mathrm{P}=0.80$, that for $\mathrm{DBP}$ was $0.35(95 \mathrm{Cl} \%:-0.39,1.10)$ with $\mathrm{I}^{2}=17.8 \%$ and $\mathrm{P}=0.25$, that for PP was $0.88(95 \mathrm{Cl} \%:-0.47,2.23)$ with $\mathrm{I}^{2}=36.4 \%$ and $\mathrm{P}=0.07$, and that for MAP was 0.69 (95 $C l \%: 0.02,1.36)$ with $\mathrm{I}^{2}=0$ and $\mathrm{P}=0.98$. Because of sample size limitations, we did not run provincespecific models for Beijing, Guangdong, Heilongjiang, Shanghai, or Zhejiang.

\section{Discussion}

\section{Summary of findings}


The results of our cross-sectional study of 8067 elderly people over 65 in 500 urban and rural communities in 23 provinces in China showed that people who used solid fuel for cooking were at a greater risk of elevated BP than those who used clean fuel. However, we did not find any significant association between solid fuel use for cooking and hypertension.

\section{Robustness of findings}

Our findings verified existing evidence that the elderly use of solid fuel for cooking is positively correlated with BP. Although a large number of older people are exposed to solid fuel pollution and may experience health effects, few studies have investigated the impact of indoor air pollution caused by solid fuel use for cooking on the elderly over the age of 65 years. Further, only two studies have examined indoor solid fuel use in the elderly as related to BP, and these studies reached contradictory conclusions. A study of 3754 participants ( $\geq 45$ years old) in China showed that people cooking with solid fuel were 1.15 times more likely to suffer from hypertension (OR $=1.15,95 \% \mathrm{Cl}: 1.01,1.31)$, and had an SBP $1.10 \mathrm{mmHg}(95 \%$ Cl: 0.48, 1.72), DBP $1.02 \mathrm{mmHg}(95 \% \mathrm{Cl}: 0.61,1.43)$, and MAP $1.03 \mathrm{mmHg}(95 \% \mathrm{Cl}: 0.63,1.43)$ higher than those who cooked with clean fuel (Deng et al., 2020). Another study of 10450 participants $(\geq 65$ years old) in China did not reveal any significant association between indoor solid fuel use for cooking and BP or hypertension (Lin et al., 2021). In addition, two Urban and Rural Epidemiology (PURE) studies considered participants aged 30-70 years. One of these studies did not find that elderly people $(\geq 50$ years old) who use indoor solid fuel were more likely to suffer from hypertension or increased BP (Arku et al., 2020), whereas a subgroup analysis in the other study showed that there was no association between solid fuel use and hypertension/BP in the elderly over 60 years (Liu et al., 2021). These studies demonstrate the variability of results related to indoor solid fuel use for cooking and BP/hypertension.

These varying results can be explained by variations in elderly sample size and insufficient covariates, especially CVD risk factors. In this study, we found that, compared with those who cooked with clean fuel, those who cooked with solid fuel had a $1.87 \mathrm{mmHg}$ higher SBP (95\% Cl: 0.85, 2.89), a $0.09 \mathrm{mmHg}$ higher DBP (95\% Cl: 0.31, 1.49), a $0.97 \mathrm{mmHg}$ higher PP (95\% Cl: 0.06, 1.88), and a $1.22 \mathrm{mmHg}$ higher MAP $(95 \% \mathrm{Cl}: 0.59,1.85)$. Our findings were robust across study regions, urban/rural status, CVD risk factors, and sociodemographic characteristics. Further, our study had a large sample of older people aged greater than 65 years, in which the average age was 84.37 years.

\section{Reducing rise in BP caused by indoor solid fuel use}

This was the first elderly-based multicenter study to investigate the association between indoor solid fuel use for cooking and BP/hypertension while controlling for comprehensive factors. The large proportion of elderly people in our study had elevated BP or suffered from hypertension may be the result of a lack of medical care. Model 3 considered education and family income, which may be a component of health care, and revealed the weakest impact of solid fuel on BP of all the models used. Specifically, the SBP, DBP, PP, and MAP values decreased from $2.72 \mathrm{mmHg}(95 \% \mathrm{Cl}: 1.73,3.70), 1.63 \mathrm{mmHg}(95 \% \mathrm{Cl}: 1.06$, 2.20), $1.09 \mathrm{mmHg}$ (95\% Cl: $0.21,1.96)$, and $1.99 \mathrm{mmHg}(95 \% \mathrm{Cl}: 1.38,2.60)$ in Model 2 to $1.87 \mathrm{mmHg}$ (95\% Cl: $0.85,2.89), 0.90$ mmHg (95\% Cl: 0.31, 1.49), 0.97 mmHg (95\% Cl: $0.06,1.88)$, and $1.22 \mathrm{mmHg}$ 
(95\% Cl: $0.59,1.85)$ in Model 3, respectively, which are consistent with the large PURE study (Hystad et al., 2019). Although we did not observe a significant positive association between indoor solid fuel use and hypertension in Model 3, our study included approximately 500 sample areas in 23 provinces in China, which may not be representative of all of China.

\section{Elderly over 75 years old prioritize replacing indoor solid fuel}

Our study provided a novel finding that long-livers $(b=1.94 \mathrm{mmHg}, 95 \% \mathrm{Cl}: 0.32,3.56$ for SBP; $b=1.18$ $\mathrm{mmHg}, 95 \% \mathrm{Cl}: 0.16,2.20$ for MAP) were at greater risk of increasing BP than those of senile age among those using solid fuel for cooking ( $b=3.37 \mathrm{mmHg}, 95 \% \mathrm{Cl}: 1.57,5.17$ for SBP; $b=2.14 \mathrm{mmHg}, 95 \% \mathrm{Cl}$ : $1.06,3.22$ for MAP). Age is a determining risk associated with increased BP and hypertension (Peter $L$, 2017), and the results of the 2012-2015 Chinese Hypertension Study showed the prevalence of hypertension was $55.7 \%$ in those aged $65-74$ years, and $60.2 \%$ in those aged $\geq 75$ years (Wang et al., 2018). Therefore, with increasing population life expectancy, the number of elderly hypertensive patients is also expected to increase. These results provide useful insights for countries that are experiencing an aging society like China. In the case of limited clean energy facilities or other human and material resources, priority should be given to the susceptible population, which is those aged over 75 years.

\section{Different groups should give different attention}

According to the stratified analyses, using indoor solid fuel for cooking by northern residents had a greater impact on BP than that for southern residents. One possible explanation for this is that the longterm exposure of northern residents to $\mathrm{PM}_{2.5}$, combined with the harmful gases released by using indoor solid fuel, increased the BP of northern residents (Dong et al., 2013; Li et al., 2018). Note that we found a significant association for women over the age of 65 using solid fuel for cooking, but not in men. In most Chinese households, women are the primary cooks, meaning that elderly women are exposed to air pollution for a longer time than men, giving them higher exposure levels (Austin and Mejia, 2017; Ofori et al., 2018). As the results showed, the elderly over the age of 75 were most affected by solid fuel use. In addition, the BP of the elderly suffering from hypertension and heart disease was significantly related to the use of indoor solid fuel, which is consistent with some previous studies (Dutta et al., 2011; Misun L, 2012), but contrary to others (Arku et al., 2020). We also found that natural ventilation had the greatest impact on BP. However, we did not observe any association with mechanical ventilation and no ventilation. This is likely because there were only 696 no ventilation cases documented in the data, and mechanical ventilation had the best ventilation effect. The magnitude of these differences was consistent with previous findings concerning the impact of ventilation (EA Rehfuess, 2011).

\section{Strengths and limitations}

Our study has several strengths, including a large sample size from 500 urban and rural communities across 23 provinces in China, making it a useful supplement to the elderly group data for future studies (Arku et al., 2020; Liu et al., 2021). Further, the inclusion of multiple provinces and communities increased the generality of the results, the extensive individual information collected aided to control 
confusion, and standardizing and defining comprehensive and systematic information helped to evaluate the results. However, there were also some limitations. First, we had no information regarding whether the individuals were responsible for cooking, what types of cooking they experienced during their childhood, and how much time they spent cooking with indoor solid fuel. In addition, indoor air pollution exposure may vary as a result of familial and personal characteristics (Balakrishnan et al., 2011). Second, we did not have the capacity to evaluate specific fuel types (for example, manure, wood, and agricultural products), and additional analyses are required in future research. Third, we used indoor solid fuel for cooking as the only indicator of indoor air pollution exposure because we did not know the exact residential addresses of participants; therefore, we did not collect personal level exposure $\mathrm{PM}_{2.5}$ data (Adar et al., 2018; Zhang et al., 2019). Fourth, our study did not include temperature and humidity, both of which impact BP and hypertension (Lewington et al., 2012; Modesti, 2013), because the long-term temperature and humidity of the family were not measured in the survey.

\section{Conclusions}

We found that, compared with households that use clean fuel for cooking, elderly persons living in households that use indoor solid fuel for cooking had an increased risk of elevated BP. The results from this large, diverse population of 8067 elderly people aged over 65 years from 500 urban and rural communities in 23 provinces in China supports the evidence that solid fuel use for cooking is positively related to BP. On this basis, replacing solid fuel for cooking with clean fuel may be an important way to lower BP, and the most susceptible population, those aged over 75 years, northern residents, women, and hypertensive and heart patients, should be given priority access to clean fuels.

\section{Declarations}

\section{Acknowledgment}

Thanks to the Chinese Longitudinal Healthy Longevity Survey team for providing data.

\section{Competing Interests}

The authors declared that they have no conflict of interest.

\section{Funding}

This study was supported by National Natural Science Foundation of China (71774102).

\section{Author contributions}

Qiutong Yu contributed to conceptualization, data curation, formal analysis, methodology, writing, visualization and editing. Genyong Zuo contributed to conceptualization, methodology, editing. All authors read and approved the final version to be published. 


\section{Data available statement}

Data were available in open research data service platform of Peking University. Data for this study were sourced from Chinese Longitudinal Healthy Longevity Survey (CLHLS) and available here: https://opendata.pku.edu.cn

\section{Ethics approval and consent to participate}

The CLHLS was approved by the Ethical Review Committee of Peking University (IRB00001052-13074). All participants signed the informed consent at the time of participation. The research has been performed in accordance with the Declaration of Helsinki.

\section{Oricd}

Qiutong Yu http://orcid.org/0000-0002-1758-0692

Genyong Zuo http://orcid.org/0000-0002-9305-9671

\section{References}

1. Adar SD, Chen YH, D'Souza JC, O'Neill MS, Szpiro AA, Auchincloss AH, Park SK et al (2018) Longitudinal analysis of long-term air pollution levels and blood pressure: A cautionary tale from the multi-ethnic study of atherosclerosis. Environ Health Perspect. 126,107003.https://doi.org/10.1289/EHP2966.

2. Arku RE, Ezzati M, Baumgartner J, Fink G, Zhou B, Hystad P, Brauer M (2018) Elevated blood pressure and household solid fuel use in premenopausal women: Analysis of 12 demographic and health surveys (dhs) from 10 countries. Environ Res. 160,499-

505.https://doi.org/10.1016/j.envres.2017.10.026.

3. Arku RE, Brauer M, Ahmed SH, AlHabib KF, Avezum A, Bo J, Choudhury T et al (2020) Long-term exposure to outdoor and household air pollution and blood pressure in the prospective urban and rural epidemiological (pure) study. Environ Pollut. 262,114197.https://doi.org/10.1016/j.envpol.2020.114197.

4. Austin KF, Mejia MT (2017) Household air pollution as a silent killer: Women's status and solid fuel use in developing nations. Population and Environment. 39,1-25.https://doi.org/10.1007/s11111017-0269-z.

5. Balakrishnan K, Ramaswamy P, Sambandam S, Thangavel G, Ghosh S, Johnson P, Mukhopadhyay K et al (2011) Air pollution from household solid fuel combustion in india: An overview of exposure and health related information to inform health research priorities. Glob Health Action. 4https://doi.org/10.3402/gha.v4i0.5638.

6. Bonjour S, Adair-Rohani H, Wolf J, Bruce NG, Mehta S, Pruss-Ustun A, Lahiff M et al (2013) Solid fuel use for household cooking: Country and regional estimates for 1980-2010. Environ Health Perspect. 
121,784-790.https://doi.org/10.1289/ehp.1205987.

7. Brauer M, Casadei B, Harrington RA, Kovacs R, Sliwa K, Group WHFAPE (2021) Taking a stand against air pollution-the impact on cardiovascular disease: $A$ joint opinion from the world heart federation, american college of cardiology, american heart association, and the european society of cardiology. Circulation. 143,e800-e804.https://doi.org/10.1161/CIRCULATIONAHA.120.052666.

8. Chittaranjan S Yajnik JSY (2004) Appropriate body-mass index for asian populations and its implications for policy and intervention strategies. The Lancet. 363,157-

163.https://doi.org/10.1016/s0140-6736(03)15268-3.

9. Chobanian AV, Bakris GL, Black HR, Cushman WC, Green LA, Izzo JL, Jr., Jones DW et al (2003) Seventh report of the joint national committee on prevention, detection, evaluation, and treatment of high blood pressure. Hypertension. 42,1206-

1252.https://doi.org/10.1161/01.HYP.0000107251.49515.c2.

10. Cohen AJ, Brauer M, Burnett R, Anderson HR, Frostad J, Estep K, Balakrishnan K et al (2017) Estimates and 25-year trends of the global burden of disease attributable to ambient air pollution: An analysis of data from the global burden of diseases study 2015 . The Lancet. 389,19071918.https://doi.org/10.1016/s0140-6736(17)30505-6.

11. Deng Y, Gao Q, Yang D, Hua H, Wang N, Ou F, Liu R et al (2020) Association between biomass fuel use and risk of hypertension among chinese older people: A cohort study. Environ Int. 138,105620.https://doi.org/10.1016/j.envint.2020.105620.

12. Dong GH, Qian ZM, Xaverius PK, Trevathan E, Maalouf S, Parker J, Yang L et al (2013) Association between long-term air pollution and increased blood pressure and hypertension in china. Hypertension. 61,578-584.https://doi.org/10.1161/HYPERTENSIONAHA.111.00003.

13. Dutta A, Mukherjee B, Das D, Banerjee A, Ray MR (2011) Hypertension with elevated levels of oxidized low-density lipoprotein and anticardiolipin antibody in the circulation of premenopausal indian women chronically exposed to biomass smoke during cooking. Indoor Air. 21,165176.https://doi.org/10.1111/j.1600-0668.2010.00694.x.

14. EA Rehfuess NB, KR Smith (2011) Solid fuel use: Health effect. Encyclopedia of Environmental Health.150-161.

15. Everson F, De Boever P, Nawrot TS, Goswami N, Mthethwa M, Webster I, Martens DS et al (2019) Personal no2 and volatile organic compounds exposure levels are associated with markers of cardiovascular risk in women in the cape town region of south africa. Int J Environ Res Public Health. 16https://doi.org/10.3390/ijerph16132284.

16. Fatmi Z, Ntani G, Coggon D (2019) Coronary heart disease, hypertension and use of biomass fuel among women: Comparative cross-sectional study. BMJ Open. 9,e030881.https://doi.org/10.1136/bmjopen-2019-030881.

17. Gall ET, Carter EM, Earnest CM, Stephens B (2013) Indoor air pollution in developing countries: Research and implementation needs for improvements in global public health. Am J Public Health. 103,e67-72.https://doi.org/10.2105/AJPH.2012.300955. 
18. Gina K TB, Marie C(2010) Guidelines for measuring household and individual dietary diversity. Nutrition and Consumer Protection Division, Food and Agriculture Organization of the United Nations ed.

19. Howard D. Sesso MJS, Bernard Rosner, Charles H. Hennekens, J. Michael Gaziano, JoAnn E. Manson, Robert J. Glynn (2000) Systolic and diastolic blood pressure, pulse pressure, and mean arterial pressure as predictors of cardiovascular disease risk in men. Hypertension. 36,801-807.

20. Hystad P, Duong M, Brauer M, Larkin A, Arku R, Kurmi OP, Fan WQ et al (2019) Health effects of household solid fuel use: Findings from 11 countries within the prospective urban and rural epidemiology study. Environ Health Perspect. 127,57003.https://doi.org/10.1289/EHP3915.

21. IHME (2019a) Cardiovascular diseases - level 2 cause. http://www.healthdata.org/results/gbd_summaries/2019/high-systolic-blood-pressure-level-2risk., Access date: 9 September 2021.

22. IHME (2019b) Cardiovascular diseases - level 2 cause. 2019. http://www.healthdata.org/results/gbd_summaries/2019/cardiovascular-diseases-level-2cause, Access date: 9 September 2021.

23. Jackson T. Wright J, George Bakris, Tom Greene (2002) African american study of kidney disease and hypertension study group. Effect of blood pressure lowering and antihypertensive drug class on progression of hypertensive kidney disease. JAMA. 288,2421-2431.

24. Ji JS (2018) Air pollution and china's ageing society. The Lancet Public Health. 3,e457e458.https://doi.org/10.1016/s2468-2667(18)30179-8.

25. Khan JR, Hossain MB, Gupta RD (2021) Household cooking fuels associated with elevated blood pressure among adult women: A national-wide assessment in bangladesh. Environ Sci Pollut Res Int.https://doi.org/10.1007/s11356-021-15344-w.

26. Lewington S, Li L, Sherliker P, Guo Y, Millwood I, Bian Z, Whitlock G et al (2012) Seasonal variation in blood pressure and its relationship with outdoor temperature in 10 diverse regions of china: The china kadoorie biobank. J Hypertens. 30,1383-

1391.https://doi.org/10.1097/HJH.0b013e32835465b5.

27. Li T, Zhang Y, Wang J, Xu D, Yin Z, Chen H, Lv Y et al (2018) All-cause mortality risk associated with long-term exposure to ambient pm2.5 in china: A cohort study. The Lancet Public Health. 3,e470e477.https://doi.org/10.1016/s2468-2667(18)30144-0.

28. Lim SS, Vos T, Flaxman AD, Danaei G, Shibuya K, Adair-Rohani H, AlMazroa MA et al (2012) A comparative risk assessment of burden of disease and injury attributable to 67 risk factors and risk factor clusters in 21 regions, 1990-2010: A systematic analysis for the global burden of disease study 2010. The Lancet. 380,2224-2260.https://doi.org/10.1016/s0140-6736(12)61766-8.

29. Lin L, Wang HH, Liu Y, Lu C, Chen W, Guo VY (2021) Indoor solid fuel use for heating and cooking with blood pressure and hypertension: A cross-sectional study among middle-aged and older adults in china. Indoor Air. 00,1-9.https://doi.org/10.1111/ina.12872. 
30. Liu C, Chen R, Zhao Y, Ma Z, Bi J, Liu Y, Meng X et al (2017) Associations between ambient fine particulate air pollution and hypertension: A nationwide cross-sectional study in china. Sci Total Environ. 584-585,869-874.https://doi.org/10.1016/j.scitotenv.2017.01.133.

31. Liu Z, Hystad P, Zhang Y, Rangarajan S, Yin L, Wang Y, Hu B et al (2021) Associations of household solid fuel for heating and cooking with hypertension in chinese adults. J Hypertens. 39,667676.https://doi.org/10.1097/HJH.0000000000002689.

32. Misun L JH, Fengying Z, Helian D (2012) In-home solid fuel use and cardiovascular disease: A crosssectional analysis of the shanghai putuo study. Environmental Health. 11,18.

33. Modesti PA (2013) Season, temperature and blood pressure: A complex interaction. Eur J Intern Med. 24,604-607.https://doi.org/10.1016/j.ejim.2013.08.002.

34. National Bureau of Statistics of China (2021) Interpretation of the bulletin of the seventh national census. http://www.stats.gov.cn/tjsj/sjjd/202105/t20210512_1817336.html, Access date: 9 September 2021.

35. Ofori SN, Fobil JN, Odia OJ (2018) Household biomass fuel use, blood pressure and carotid intima media thickness; a cross sectional study of rural dwelling women in southern nigeria. Environ Pollut. 242,390-397.https://doi.org/10.1016/j.envpol.2018.06.102.

36. Peter L MG, Paul O(2017) Hypertension and older people. HelpAge International ed.

37. Prusinowska A, Komorowski A, Przepiora W, Ksiezopolska-Orlowska K (2016) Physical activity in the elderly who underwent joint replacement surgery in the course of rheumatic diseases. Reumatologia. 54,117-121.https://doi.org/10.5114/reum.2016.61211.

38. R Collins RP, S MacMahon,J Godwin, N Qizilbash, MRCP, R Collins (1990) Blood pressure, stroke, and coronary heart disease: Part 2, short-term reductions in blood pressure. Lancet. 335,827-838.

39. Stanaway JD, Afshin A, Gakidou E, Lim SS, Abate D, Abate KH, Abbafati C et al (2018) Global, regional, and national comparative risk assessment of 84 behavioural, environmental and occupational, and metabolic risks or clusters of risks for 195 countries and territories, 1990-2017: A systematic analysis for the global burden of disease study 2017. The Lancet. 392,19231994.https://doi.org/10.1016/s0140-6736(18)32225-6.

40. Wang L, Xiang Z, Stevanovic S, Ristovski Z, Salimi F, Gao J, Wang H et al (2017) Role of chinese cooking emissions on ambient air quality and human health. Sci Total Environ. 589,173181.https://doi.org/10.1016/j.scitotenv.2017.02.124.

41. Wang Z, Chen Z, Zhang L, Wang X, Hao G, Zhang Z, Shao L et al (2018) Status of hypertension in china: Results from the china hypertension survey, 2012-2015. Circulation. 137,23442356.https://doi.org/10.1161/CIRCULATIONAHA.117.032380.

42. World Health Organization (2013) A global brief on hypertension : Silent killer, global public health crisis: World health day 2013. https://apps.who.int/iris/handle/10665/79059, Access date: 30 September 2021.

43. World Health Organization (2021) Ambient air pollution. https://www.who.int/teams/environmentclimate-change-and-health/air-quality-and-health/ambient-air-pollution, Access date: 9 September 
2021.

44. Yan M, Li C, Zhang L, Chen X, Yang X, Shan A, Li X et al (2020) Association between long-term exposure to sulfur dioxide pollution and hypertension incidence in northern china: A 12-year cohort study. Environ Sci Pollut Res Int. 27,21826-21835.https://doi.org/10.1007/s11356-020-08572-z.

45. Yan Z, Liu Y, Yin Q, Qiu M (2016) Impact of household solid fuel use on blood pressure and hypertension among adults in china. Air Quality, Atmosphere \& Health. 9,931940.https://doi.org/10.1007/s11869-016-0395-2.

46. Yang BY, Guo Y, Morawska L, Bloom MS, Markevych I, Heinrich J, Dharmage SC et al (2019) Ambient pm1 air pollution and cardiovascular disease prevalence: Insights from the 33 communities chinese health study. Environ Int. 123,310-317.https://doi.org/10.1016/j.envint.2018.12.012.

47. Yao Y, Jin X, Cao K, Zhao M, Zhu T, Zhang J, Zeng Y (2021) Residential proximity to major roadways and cognitive function among chinese adults 65 years and older. Sci Total Environ. 766,142607.https://doi.org/10.1016/j.scitotenv.2020.142607.

48. Yu K, Qiu G, Chan KH, Lam KH, Kurmi OP, Bennett DA, Yu C et al (2018) Association of solid fuel use with risk of cardiovascular and all-cause mortality in rural china. JAMA. 319,13511361.https://doi.org/10.1001/jama.2018.2151.

49. Zhang Z, Dong B, Li S, Chen G, Yang Z, Dong Y, Wang Z et al (2019) Exposure to ambient particulate matter air pollution, blood pressure and hypertension in children and adolescents: A national crosssectional study in china. Environ Int. 128,103-108.https://doi.org/10.1016/j.envint.2019.04.036.

\section{Figures}

(A)

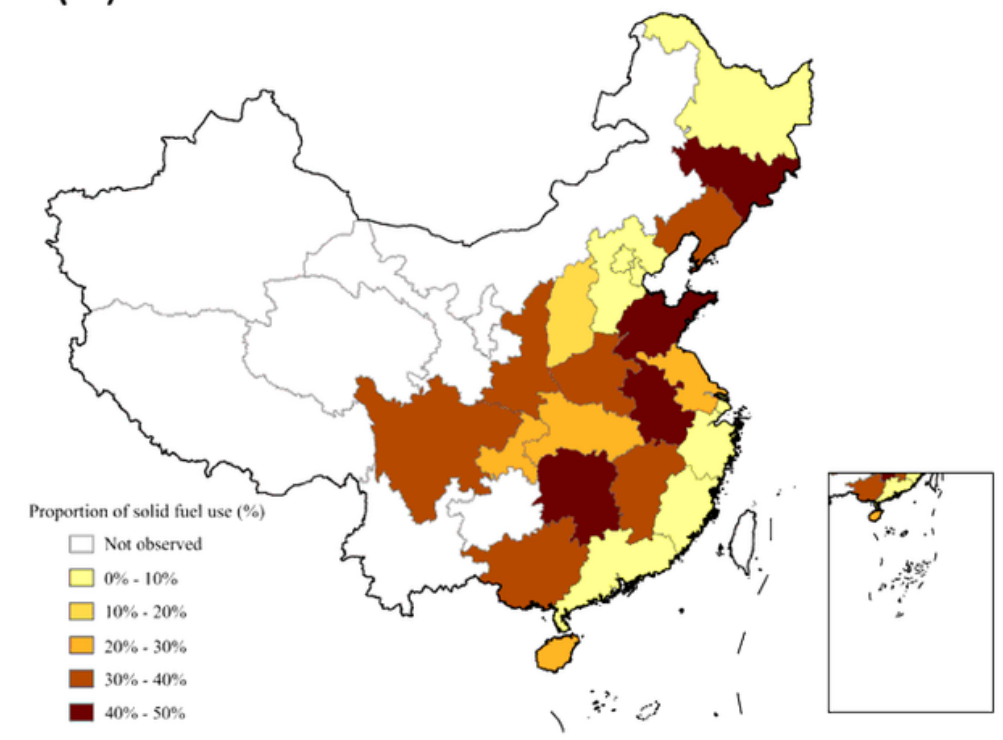

(B)

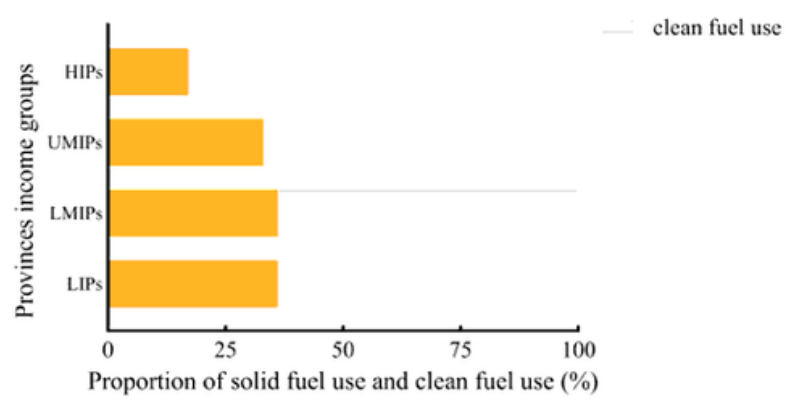

Figure 1 
Proportion of solid fuel used by province of China based on 2018 CLHLS (A); Percentage chart shows the proportion of solid fuel use classified by per capita disposable income of provinces (B). High-income provinces (HIPs): Beijing, Jiangsu, Shanghai, Tianjin, Zhejiang; Upper Middle-income provinces (UMIPs): Fujian, Guangdong, Liaoning, Shandong, Chongqing; Lower Middle-income provinces (LMIPs): Anhui, Hainan, Hebei, Heilongjiang, Hubei, Hunan, Jilin, Jiangxi, Shaanxi; Low-income provinces (LIPs): Guangxi, Henan, Shanxi, Sichuan. The economic level classification of a province is based on the interquartile range of the per capita disposable income of each province released by the National Bureau of Statistics in 2018.

(A)

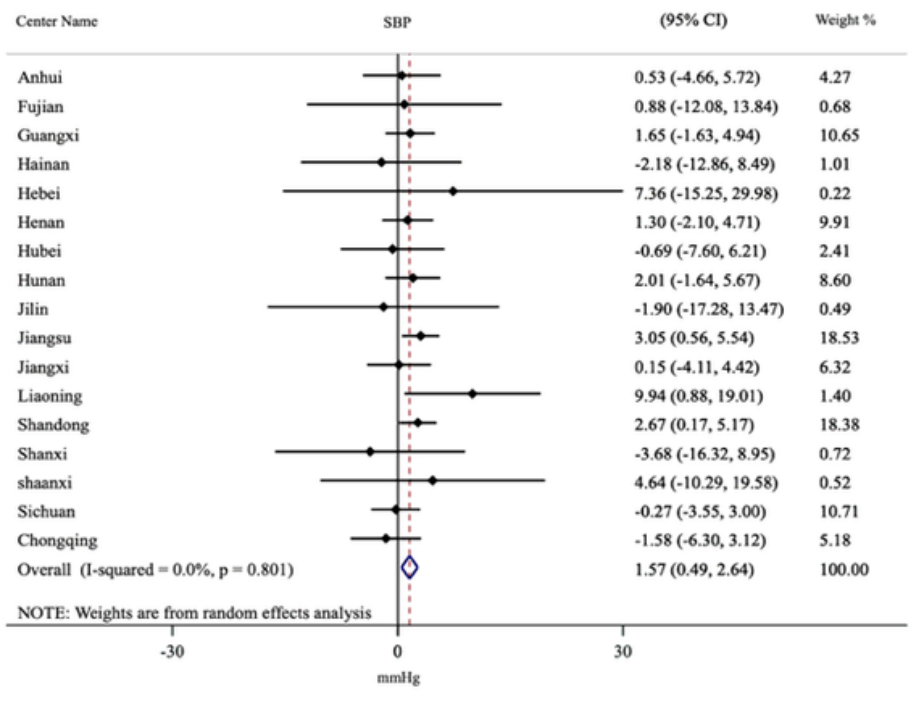

(C)

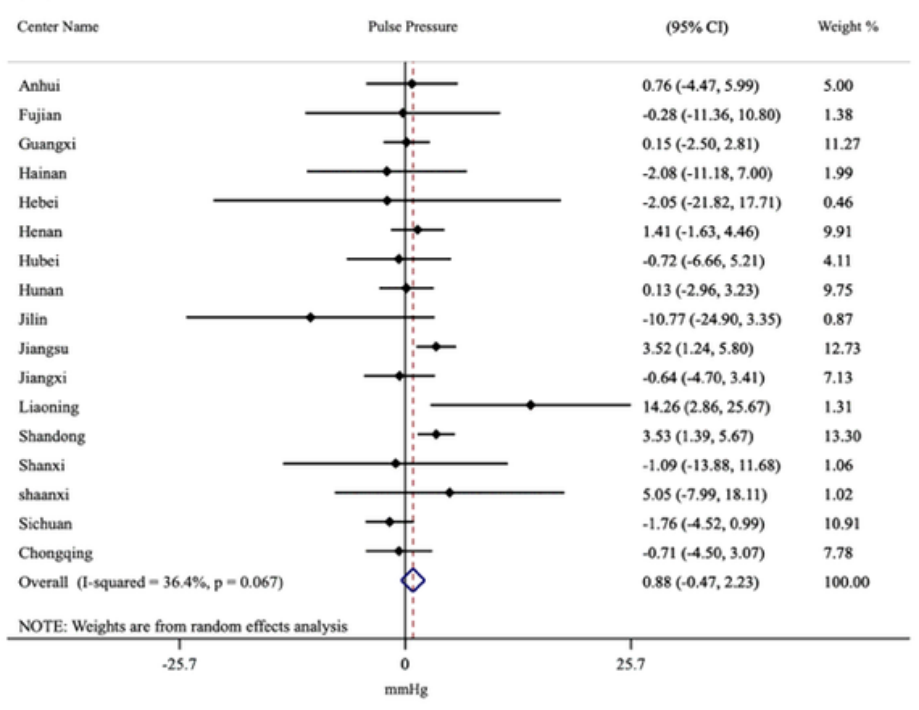

(B)

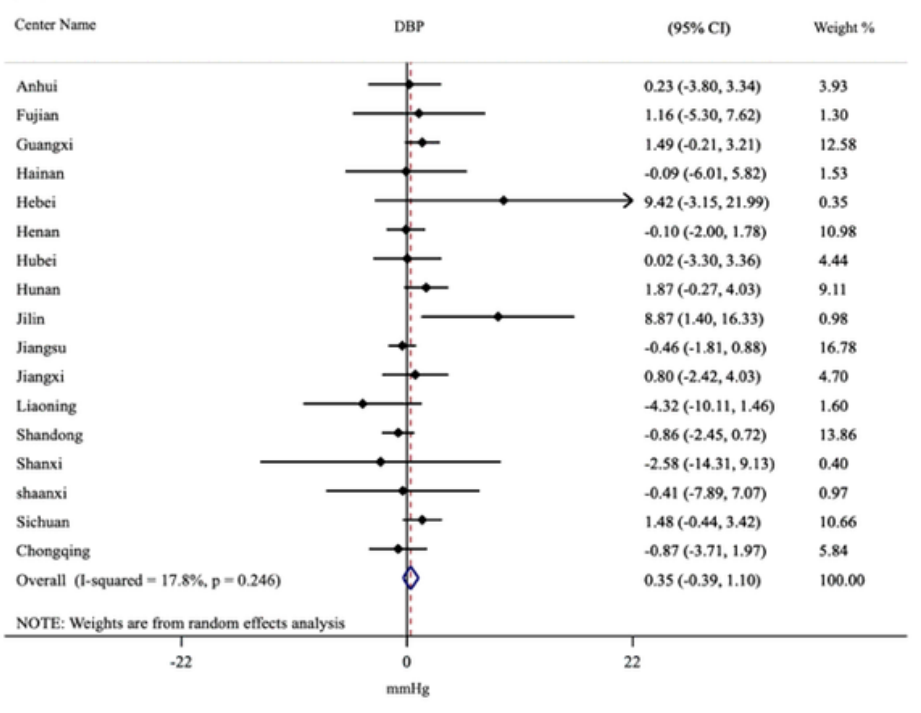

(D)

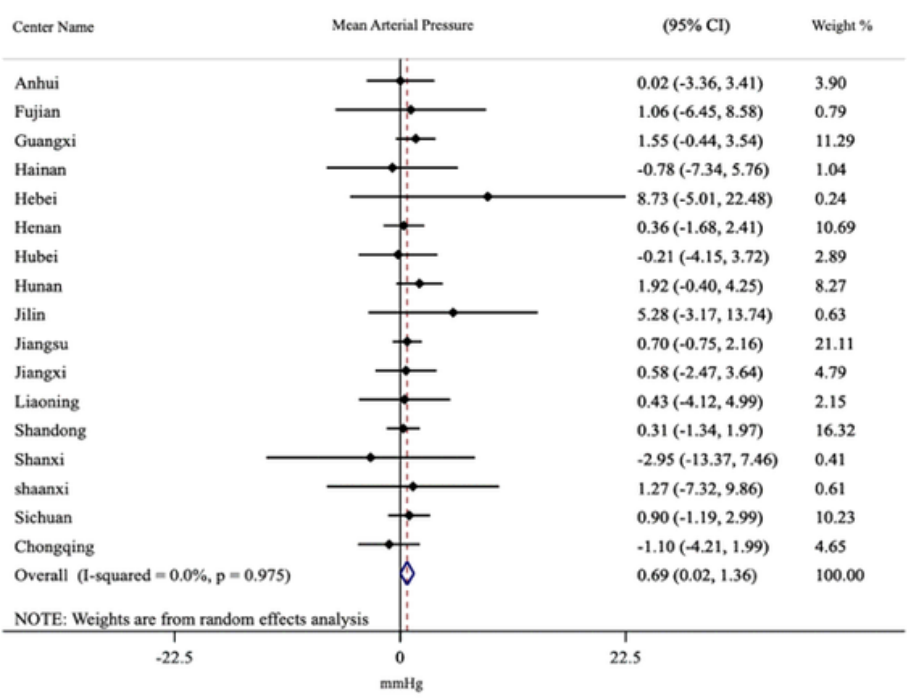

Figure 2

Model estimates of multivariable, province-specific, and meta-analysis of the association of solid fuel use with (A) SBP; (B) DBP; (C) PP; and (D) MAP based on 2018 CLHLS. 


\section{Supplementary Files}

This is a list of supplementary files associated with this preprint. Click to download.

- Onlinefloatimage3.png 